.

\title{
Conocimiento sobre los dispositivos electrónicos de entrega de nicotina en médicos residentes de neumología en un centro de referencia
}

\section{Understanding of electronic nicotine delivery systems in resident physicians in a reference center of pulmonary diseases}

\author{
Carla Paola Sánchez-Ríos, Jerónimo Rafael Rodríguez-Cid,* Luis Manuel Martínez-Barrera,* \\ Patricio Santillán-Doherty, Jorge Arturo Alatorre-Alexander*
}

*Instituto Nacional de Enfermedades Respiratorias Ismael Cosío Villegas, Ciudad de México.

\begin{abstract}
RESUMEN. Introducción: El tabaquismo sigue siendo un problema de salud pública. Se considera la causa principal de muerte prevenible en el mundo. En nuestro país, a pesar de las políticas antitabaco, la falta de regulación del comercio de los dispositivos electrónicos de entrega de nicotina y su uso en la población de jóvenes escolares continúa siendo un problema debido a la desinformación sobre los riesgos a la salud a corto y mediano plazo, además de que aumentan el riesgo de consumo en los fumadores que lo usan como terapia para dejar de fumar, y de llegar a caer en el consumo de tabaco convencional en aquéllos no fumadores. Objetivo: Describir el conocimiento de los médicos residentes de neumología sobre los dispositivos electrónicos de entrega de nicotina. Material y métodos: Estudio observacional descriptivo y transversal sobre el conocimiento de los dispositivos electrónicos de entrega de nicotina en médicos residentes de neumología. Se aplicó una encuesta con 15 ítems de opción múltiple a 30 médicos residentes de neumología. Los resultados se evaluaron y expresaron mediante medidas de tendencia central. Resultados: Se encontró que $60 \%$ de los médicos tenía conocimiento de los dispositivos electrónicos de entrega de nicotina; sólo 66.67\% tenía conocimiento sobre el funcionamiento; $90 \%$ no lo recomienda debido a la falta de eficacia y $90 \%$ comentó desconocer cuestiones legales; $50 \%$ desconoce información sobre la seguridad y $86.67 \%$ desconoce información sobre costos; $90 \%$ consideró necesitar mayor información sobre los dispositivos electrónicos de entrega de nicotina. Conclusiones: Se demuestra la clara necesidad de mejorar el conocimiento de los dispositivos electrónicos de entrega de nicotina en médicos neumólogos en formación.
\end{abstract}

Palabras clave: Dispositivos electrónicos de entrega de nicotina, deshabituación tabáquica, seguridad.

Correspondencia:

Dra. Carla Paola Sánchez-Ríos

Instituto Nacional de Enfermedades Respiratorias

Ismael Cosío Villegas, Ciudad de México.

Correo electrónico: pao1144tost@gmail.com

Trabajo recibido: 06-VIII-2019; aceptado: 14-II-2020.

Citar como: Sánchez-Ríos CP, Rodríguez-Cid JR, Martínez-Barrera LM, Santillán-Doherty P, Alatorre-Alexander JA. Conocimiento sobre los dispositivos electrónicos de entrega de nicotina en médicos residentes de neumología en un centro de referencia. Neumol Cir Torax. 2020;79(3):164-170. https:// dx.doi.org/10.35366/96651
ABSTRACT. Introduction: Smoking remains a public health problem. It is considered the leading cause of preventable death in the world. In our country, despite anti-tobacco policies, the lack of regulation of trade in ENDS (Electronic Nicotine Delivery Systems) and its use in the population of young schoolchildren due to lack of information about short-term health risks remains a problem and medium term and that, increase the risk of continuing to smoke in smokers who use it as a smoking cessation therapy and of falling into conventional tobacco use in non-smokers. Objective: To describe the knowledge of the resident physicians of pulmonology about the END. Material and methods: Prospective cross-sectional observational study on the knowledge of END in pulmonary residents, a survey with 15 multiple-choice items was applied. The results were evaluated and expressed using measures of central tendency. Results: It was found that $60 \%$ of the doctors had knowledge of END, only $66.67 \%$ had knowledge about the functioning. $90 \%$ do not recommend it due to lack of effectiveness and $90 \%$ said they ignore legal issues. $50 \%$ do not know information about security, $86.67 \%$ do not know about cost information. $90 \%$ considered needing more information about the END. Conclusions: The clear need to improve the knowledge of END in medical pulmonologists in training is demonstrated.

Keywords: Electronic nicotine delivery systems, smoking cessation, security.

\section{INTRODUCCIÓN}

Los dispositivos electrónicos de entrega de nicotina (ENDS) abarcan todos los cigarrillos electrónicos (Cig-e), vapeadores electrónicos, hookahs electrónicas, pluma de vapeo y sistemas de tanques utilizados a altas temperaturas. Son todos dispositivos que funcionan con baterías, que calientan los líquidos electrónicos y suministran nicotina en aerosol, saborizantes, humectantes y otros productos químicos. Los líquidos electrónicos pueden contener altos niveles de nicotina y están disponibles en varios sabores 
que pueden atraer a los jóvenes. El uso de ENDS puede provocar la exposición a compuestos orgánicos volátiles tóxicos, metales pesados y nicotina. Dentro de éstos se encuentran los Cig-e, dispositivos que contienen un líquido que al ser calentado produce un aerosol (vapor) que es inhalado («vapeado») por el usuario.' Los Cig-e constan de cuatro partes elementales: la batería, el elemento calentador, la cámara de vaporización y el cartucho de la solución (es el que contiene el líquido que se convierte en el aerosol). El líquido consta casi siempre de un solvente que puede ser glicerina vegetal, propilenglicol o una mezcla de ellos y uno o más saborizantes. La nicotina, si bien está presente en la mayoría de los líquidos de los Cig-e, puede no encontrarse., ${ }^{2,3} \mathrm{El}$ proceso de generación del vapor del Cig-e es completamente diferente al del humo del cigarrillo convencional, ya que se efectúa por un proceso de calentamiento a diferencia del proceso de combustión que ocurre en el cigarrillo convencional. Sin embargo, esto no exenta a los Cig-e de producir carcinógenos directos sobre la vía respiratoria como anteriormente se pensaba. En el Cig-e la activación del mecanismo de calentamiento se realiza gracias a un sensor que detecta cuando el usuario realiza una bocanada o «vapeo» activando una bobina electrónica que calienta y vaporiza el líquido, creando un humo de vapor visible. En los últimos años, el uso de los Cig-e ha aumentado, en especial entre los escolares y la gente joven, habiéndose más que cuadriplicado en los últimos años. En la revisión sistemática y metaanálisis de Yoong et al. en 2018 se comenta que entre 2008 y 2015 el uso de ENDS entre los jóvenes aumentó en Polonia, Corea, Nueva Zelanda y Estados Unidos; disminuyó en Italia y Canadá; y se mantuvo estable en el Reino Unido. ${ }^{4}$

Actualmente se sabe que los Cig-e también entregan, aunque en menor medida, toxinas y potenciales carcinógenos a la vía respiratoria, aunque el efecto a largo plazo en las vías aéreas y el pulmón aún se desconoce. La cantidad de nicotina entregada por los Cig-e de últimas generaciones no es diferente a la entregada por los cigarrillos convencionales, de manera que perpetúa la adicción y el hábito de fumar. ${ }^{5}$ Hay evidencia inconsistente en relación con las modalidades más efectivas en las terapias para dejar de fumar. Se han realizado varios metaanálisis que dejan clara esta inconsistencia. Hartmann-Boyce J, et al. en 2018 reportaron en su metaanálisis que de 136 estudios que exploraban la efectividad de la terapia de reemplazo de nicotina versus placebo para dejar de fumar, el riesgo relativo de abstinencia para cualquier forma de TRN en relación con el control fue de 1.55 (intervalo de confianza [IC] de 95\%: 1.49 a 1.61) concluyendo que a pesar de los sesgos de estudios, existe evidencia de alta calidad de que todas las formas autorizadas de terapia de reemplazo de nicotina (TRN) (chicle, parche transdérmico, aerosol nasal, inhalador y tabletas/pastillas sublinguales) pueden ayudar a las personas que intentan dejar de fumar a aumentar sus posibilidades de conseguirlo, con una tasa de éxito de 50 a $60 \%$, independientemente de la configuración. ${ }^{6}$

McRobbie H, et al. reportaron en 2014 que hasta ese entonces sólo había evidencia de dos ensayos sobre el hecho de que los Cig-e ayudan a los fumadores a dejar de fumar a largo plazo en comparación con placebo; ${ }^{7}$ sin embargo, en relación con su efectividad comparada con la terapia de reemplazo de nicotina, el ensayo clínico de Hajek et al. de 2019 publicado en NEJM reportó que la tasa de abstinencia a un año fue de $18.0 \%$ en el grupo de Cig-e, en comparación con 9.9\% en el grupo de reemplazo de nicotina (riesgo relativo, 1.83; IC 95\%, 1.30 a 2.58; $\mathrm{p}<0.001$ ), además de que este grupo reportó mayores disminuciones en la incidencia de tos y producción de flema desde el inicio hasta las 52 semanas que el grupo de reemplazo de nicotina (riesgo relativo de tos, 0.8 ; IC 95\%, 0.6 a 0.9; riesgo relativo de flema, 0.7 ; IC 95\%, 0.6 a 0.9). ${ }^{8}$ Claro está que la gran cantidad de sesgos y errores metodológicos aquí comentados y sus conclusiones fueron debatidos por otros autores que incluso publicaron «cartas al editor» respecto a este estudio.

Livingstone-Banks J, et al. reportaron en 2019 que las intervenciones conductuales que enseñan a las personas a reconocer situaciones de alto riesgo de recaída, junto con las estrategias para hacerles frente, no proporcionaron ningún beneficio valioso para prevenir la recaída en abstemios asistidos, aunque la heterogeneidad estadística inexplicada significa que sólo estamos moderadamente seguros de esto. En las personas que dejaron de fumar con éxito usando farmacoterapia, hubo resultados mixtos en cuanto a extender la farmacoterapia por más tiempo de lo normal. El tratamiento extendido con vareniclina ayudó a prevenir la recaída, no así con tratamiento extendido con bupropión. ${ }^{9}$ Moyo F, et al. reportaron en 2018 también que la evidencia es no concluyente para apoyar la efectividad definitiva de las ayudas no farmacológicas para la toma de decisiones en terapias para dejar de fumar a largo plazo o de manera sostenida. ${ }^{10}$

En este estudio se explora el conocimiento actual de los médicos residentes de neumología de un tercer nivel de atención en México sobre ENDS.

\section{MATERIAL Y MÉTODOS}

El estudio se realizó en el Instituto Nacional de Enfermedades Respiratorias (INER) Ismael Cosío Villegas, Ciudad de México en el mes de junio de 2019 a los médicos residentes de neumología de adultos. Se aplicó una encuesta con ítems sobre el conocimiento de los dispositivos electrónicos de entrega de nicotina conocidos como ENDS, por sus siglas en inglés (Electronic Nicotine Delivery Systems). El cuestionario fue generado por grupos de interés (compuestos por dos 
médicos neumólogos, dos neumooncólogos y un oncólogo médico) y se evaluó su validez de constructo y contenido aplicándolo a un grupo reducido de médicos residentes antes de su aplicación al total de la muestra (Anexo 1). El cuestionario presencial incluyó 15 ítems en su mayoría de contestación en formato de opción múltiple que contenía preguntas relacionadas al conocimiento de la existencia de ENDS, funcionamiento, seguridad, costos, eficacia y su utilidad como parte de la terapia de deshabituación tabáquica. Se incluyeron todos los médicos residentes de neumología, adultos que se encontraban realizando rotación en el INER en el período del estudio, no se tuvieron criterios de exclusión, se eliminaron cuestionarios incompletos en el rubro de grado y/o más de dos ítems sin respuesta.

Los resultados fueron analizados mediante estadística descriptiva y expresados en términos de medidas de tendencia central.

\section{RESULTADOS}

De un total de 54 médicos residentes de primero a cuarto grado de neumología clínica invitados a participar, un total de 30 médicos residentes contestaron la encuesta.

De los médicos encuestados las distribuciones por años fueron las siguientes: residente de primer año $23.33 \%$ $(n=7)$; segundo año $16.67 \%(n=5)$; tercer año $26.66 \%$ $(n=8)$ y cuarto año $33.34 \%(n=10) ; 73.33 \%(n=22)$ de los médicos refirió el uso de consejo breve para dejar de fumar en todas las citas médicas de atención asistencial a sus pacientes fumadores; $26.67 \%(n=8)$ de los médicos que no aconseja en su práctica clínica asistencial, refirió no hacerlo por «no atender consulta médica de especialidad para deshabituación de tabaco» por el grado de residencia médica en el que se encontraba; 53.33\% $(n=16)$ contestó afirmativamente a la aseveración del conocimiento acerca de la terapia más efectiva demostrada científicamente hasta el momento para deshabituación tabáquica; sin embargo, al preguntarles cuál era el estándar de tratamiento más efectivo sólo $25 \%(n=4)$ tuvo respuesta asertiva.

$26.67 \%(n=8)$ respondieron conocer los factores predictores de éxito en un programa de deshabituación tabáquica, pero ninguno $(\mathrm{n}=0)$ tuvo respuesta asertiva; $60 \%(n=18)$ tenía conocimiento actual de la existencia de ENDS en el contexto de las múltiples probables opciones de tratamiento para deshabituación tabáquica. A la pregunta del conocimiento sobre el funcionamiento de los Cig-e $66.67 \%(n=12)$ contestó tener conocimiento. Respecto al tipo de ENDS conocidos se encontró lo siguiente: Cig-e 44.45\% ( $=7$ ), Cig-e y t-vapor 33.33\% ( $=3$ ), Cig-e, $t-$ vapor y JUUL $22.22 \%(n=2)$ (Figura 1 ).

Respecto a la pregunta relacionada con la recomendación de los médicos residentes a sus pacientes fumadores para usar ENDS como terapia para dejar de fumar, $90 \%(n=27)$ respondió que no lo recomienda y tres de ellos respondió sí recomendarlo (10\%). Cuando se les interrogó acerca de qué dispositivo tenía aprobación legal en nuestro país, 90\% $(n=27)$ respondió desconocer cuestiones legales para su comercialización en México (Figura 1). A las preguntas relacionadas con la utilidad (comparado con TRN en combinación con terapia cognitivo conductual) de esta terapia como tratamiento de deshabituación del total de residentes de primer año, 57.14\% $(n=4)$, de segundo año $60 \%(n=3)$, de tercer año $75 \%(n=6)$ y de cuarto año $60 \%(n=6)$ lo consideró una terapia no útil para ese fin (Figura 2).

Con respecto a la recomendación de cualquier tipo de ENDS en monoterapia para dejar de fumar, $83.33 \%(n=5)$ de primer año; $100 \%(n=5)$ de segundo año; $75 \%(n=6)$ de tercer año y $70 \%(n=7)$ de cuarto año no lo consideró recomendable como terapia única. El resto de cada uno de los grupos contestó sí recomendarlos en monoterapia, salvo un participante del grupo de residentes de primer año que no contestó el ítem.

Al interrogar a los médicos residentes sobre la seguridad de los ENDS, los consideraron no seguros por efectos demostrados in vitro $26.67 \%(n=8)$, los consideraron no seguros por efectos demostrados in vivo 23.33\% $(n=7) .50 \%(n=15)$ del total desconocía la información sobre la seguridad de estos dispositivos. Ningún médico residente encuestado los consideró seguros para la salud respiratoria, cardiovascular e inmunológica (Figura 3); 86.67\% $(n=26)$ del total de médicos encuestados desconocía la información sobre los costos de estos dispositivos; $90 \%(n=27)$ de los médicos residentes consideró necesitar mayor información sobre los ENDS (Figura 4).

\section{DISCUSIÓN}

Las sociedades científicas expertas en la materia de salud respiratoria: Asociación Argentina de Medicina Respiratoria (AAMR), Asociación Latinoamericana de Tórax (ALAT),

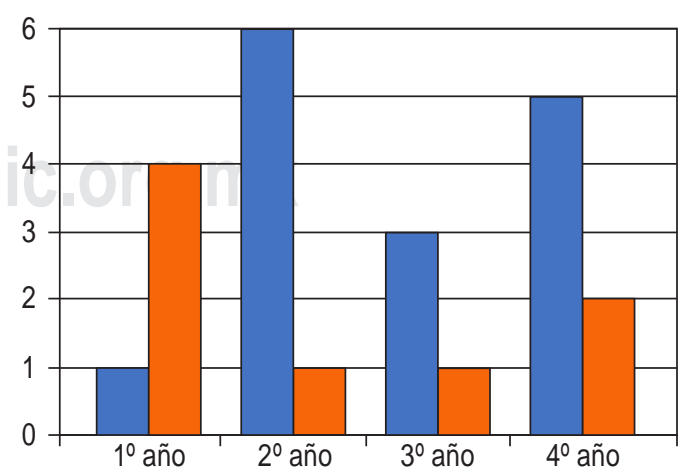

Sí conoce el funcionamiento

Figura 1: Conocimiento acerca del funcionamiento de los diferentes Cig-e en médicos residentes de Neumología, INER. 
Neumol Cir Torax. 2020; 79 (3): 164-170

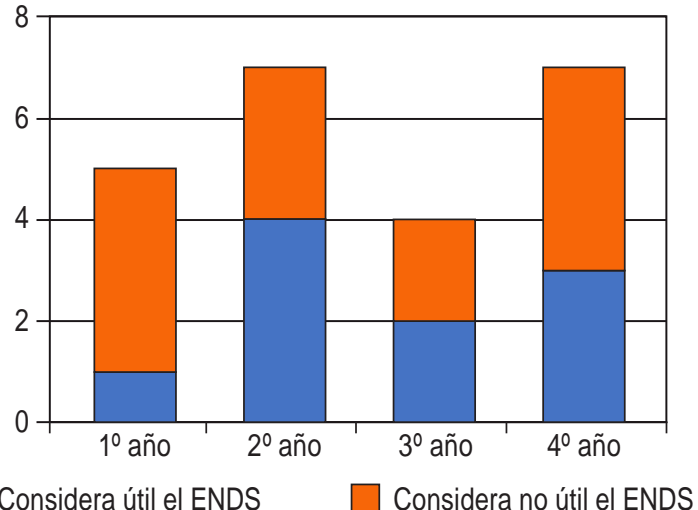

Figura 2: Conocimiento acerca de la utilidad de los ENDS como terapia de deshabituación tabáquica en médicos residentes de Neumología, INER.

Sociedad Española de Neumología y Cirugía Torácica (SEPAR), Sociedad Mexicana de Neumología y Cirugía del Tórax (SMNyCT) y European Respiratory Society (ERS) se reunieron en el «Foro de Sociedades Científicas frente al Cigarrillo Electrónico» que tuvo lugar el 2 de noviembre de 2018 en la ciudad de Buenos Aires, Argentina, durante el $46^{\circ}$ Congreso Argentino de Medicina Respiratoria y analizaron el tema emitiendo una declaración basada en el análisis de la información científica disponible, la cual se encuentra publicada, y donde se concluyó que el uso de ENDS como una estrategia de reducción de daño no puede ser apoyada porque la mayoría de los fumadores quiere abandonar el consumo de tabaco con medidas eficientes, porque el tratamiento de la adicción al tabaco actual es costo-efectivo, porque no hay pruebas que sustenten que el uso de ENDS favorece el abandono del tabaco convencional, porque no hay pruebas que sustenten que el uso de ENDS es seguro para los consumidores a largo plazo y porque aún queda una pregunta sin respuesta que es sobre la seguridad a largo plazo de cualquier forma de fumar o inhalar aerosoles que vehiculizan nicotina y finalmente, porque hay pruebas concretas que la industria tabacalera está migrando a dispositivos diferentes como las distintas formas de ENDS, manteniendo como objetivo final promover el consumo de nicotina adictiva en la población mundial y busca en los jóvenes a su clientes potenciales. ${ }^{9}$

A la fecha se sabe que no existen estudios independientes que demuestren que el Cig-e ni otros tipos de ENDS en monoterapia ayuden a dejar de fumar. En la publicación de las sociedades se hace referencia a dos metaanálisis publicados en 2014 y 2016 que concluyeron que los Cig-e no ayudan a dejar de fumar. ${ }^{10,11}$

Recientemente en febrero de 2019, NEJM publicó un ensayo clínico donde se concluyó que los Cig-e mostraron ser más efectivos que la terapia de reemplazo de nicotina (TRN) para cesación del hábito tabáquico, empero, ha re- cibido varías críticas e incluso cartas al editor en relación con la metodología del estudio. Otras investigaciones han afirmado que los usuarios de Cig-e tienen menos probabilidad de dejar el cigarrillo y que incluso favorecen el consumo dual de Cig-e y tabaco convencional, sobre todo en jóvenes, población que actualmente se considera la más vulnerable para incursionar como consumidores de tabaco..$^{12}$

Respecto a la seguridad, se han encontrado muchas sustancias tóxicas en el vapor liberado por los Cig-e y otros ENDS, entre ellas existen sustancias que pueden estar vinculadas a la activación de cascadas de carcinogénesis a nivel pulmonar y de otros órganos, además de aumentar la propensión a infecciones respiratorias por su efecto deletéreo en componentes del sistema inmunitario local y enfermedades cardiovasculares. ${ }^{13,14}$

$\mathrm{Al}$ presente se han realizado estudios en los que se demuestra el potencial dañino para el sistema respiratorio, en términos de aumento de resistencia de la vía aérea demostrada por pruebas de función respiratoria propiciando el desarrollo de enfermedades obstructivas como asma y enfermedad pulmonar obstructiva crónica que cursan con inflamación crónica de las vías aéreas.

En relación al tema de la adicción, existen pruebas contundentes y concretas de que los Cig-e aportan nicotina en niveles adictivos a sus consumidores, además la utilización de estos dispositivos ha aumentado exponencialmente entre fumadores fortaleciendo el hábito de continuar fumando. Las cantidades de nicotina no pueden regularse y no tienen una vigilancia estricta por especialistas para control de las cantidades de aporte de nicotina en estos dispositivos en el contexto de deshabituación de tabaco. , $^{15,16}$

Los expertos en salud respiratoria deberán ser los expertos en el tema con actualización científica constante y

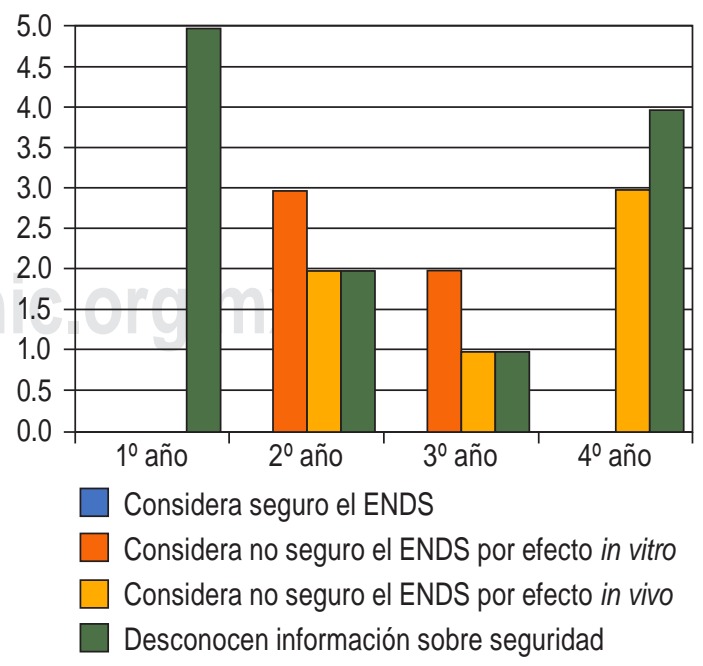

Figura 3: Conocimiento acerca de la seguridad de los ENDS como terapia de deshabituación tabáquica en médicos residentes de Neumología, INER. 


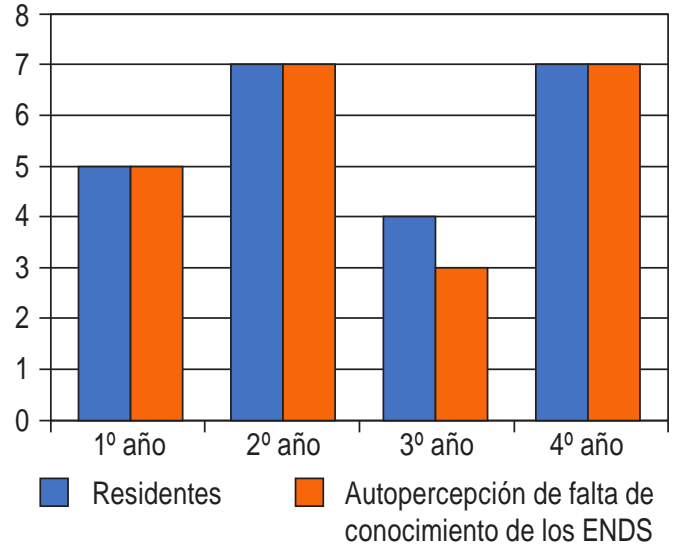

Figura 4: Autopercepción de falta de conocimiento de los ENDS y salud respiratoria en médicos residentes de Neumología, INER.

guiar e informar a los pacientes fumadores acerca de las recomendaciones actuales de ENDS en las terapias de deshabituación. Asimismo, consideramos que el tema del uso de ENDS en personas jóvenes deberá combatirse con campañas informativas acerca de las consecuencias en términos de salud del uso de estos dispositivos y del tabaco convencional cuyo consumo es un riesgo latente en quienes utilizan sistemas de vapeo con nicotina.

Por último, nos ha quedado claro que intentar prohibir el consumo de estos dispositivos mediante la falta de legalización, no ha sido una pauta efectiva para combatir el problema de su uso en nuestro país, tomando en cuenta que a pesar de que ningún tipo de ENDS tiene legalización para su comercialización, son dispositivos que se encuentran ampliamente en el mercado y que la población vulnerable consume, por lo que exhortamos a mayor cantidad de pautas y estrategias informativas, más que prohibitivas.

\section{CONCLUSIONES}

Es clara la necesidad actual de la capacitación constante de los médicos neumólogos en formación acerca de los ENDS, ya que es el personal quien deberá liderar campañas informativas a la población vulnerable (escolares y jóvenes AúN no fumadores) acerca de los riesgos a nivel de la función respiratoria que generan los dispositivos electrónicos de entrega de nicotina y el escaso beneficio (en pacientes fumadores motivados a dejar de fumar) que estos dispositivos ofrecen como tratamiento de deshabituación tabáquica.

\section{Limitaciones del estudio}

\section{Unicentro}

Cantidad reducida de la muestra (cerca de $40 \%$ de residentes inscritos en la residencia médica no participaron, por lo cual consideramos que haber realizado de manera electrónica esta encuesta pudo impactar en los resultados).

Falta de registro de variables sociodemográficas.

\section{Fortalezas}

No se han encontrado en la literatura nacional estudios similares que exploren el conocimiento de la existencia de ENDS, su funcionamiento, seguridad, costos, eficacia y utilidad como parte de la terapia de deshabituación tabáquica de médicos residentes en formación en la especialidad de neumología. Consideramos que es sumamente importante la capacitación continua de neumólogos en formación en este tema; asimismo, consideramos que al ser el INER centro de referencia que cuenta con médicos expertos en tabaco y unidad de clínica de tabaco y terapia para dejar de fumar, es un centro de alta capacitación; no obstante, hasta el momento en que se realizó dicho estudio el conocimiento de los daños y el uso expandido no había cobrado la importancia real en todo el personal involucrado como hoy en día. Los resultados nos impulsan a la capacitación continua de nuestros médicos residentes y del resto de los neumólogos en formación tanto de nuestro país como a nivel de Latinoamérica, que optan por realizar su formación de especialidad o rotaciones académicas-asistenciales en nuestro centro.

\section{REFERENCIAS}

1. Chand HS, Muthumalage T, Maziak W, Rahman I. Pulmonary toxicity and the pathophysiology of electronic cigarette, or vaping product, use associated lung injury. Front Pharmacol. 2020;10:1619. https:// doi.org/10.3389/fphar.2019.01619

2. Dinakar $\mathrm{C}, \mathrm{O}$ 'Connor GT. The health effects of electronic cigarettes. N Engl J Med. 2016;375(14):1372-1381. https://doi.org/10.1056/ nejmra1502466

3. Leduc C, Quoix E. Is there a role for e-cigarettes in smoking cessation? Ther Adv Respir Dis. 2016;10(2):130-135. https://doi. org/10.1177/1753465815621233

4. Yoong SL, Stockings E, Chai LK, Tzelepis F, Wiggers J, Oldmeadow $\mathrm{C}$, et al. Prevalence of electronic nicotine delivery systems (ENDS) use among youth globally: a systematic review and meta-analysis of country level data. Aust NZ J Public Health. 2018;42(3):303-308. https://doi.org/10.1111/1753-6405.12777

5. Orellana-Barrios MA, Payne D, Mulkey Z, Nugent K. Electronic cigarettes-a narrative review for clinicians. Am J Med. 2015;128(7):674681. https://doi.org/10.1016/i.amjmed.2015.01.033

6. Hartmann-Boyce J, Chepkin SC, Ye W, Bullen C, Lancaster T. Nicotine replacement therapy versus control for smoking cessation. Cochrane Database Syst Rev. 2018;5(5):CD000146. https://doi. org/10.1002/14651858.cd000146.pub5

7. McRobbie H, Bullen C, Hartmann-Boyce J, Hajek P. Electronic cigarettes for smoking cessation and reduction. Cochrane Database Syst Rev. 2014;(12):CD010216. https://doi.org/10.1002/14651858.cd010216.pub2

8. Hajek P, Phillips-Waller A, Przulj D, Pesola F, Smith KM, Bisal N, et al. A randomized trial of e-cigarettes versus nicotine-replacement 
therapy. N Engl J Med. 2019;380(7):629-637. https://doi.org/10.1056/ nejmoa1808779

9. Livingstone-Banks J, Norris E, Hartmann-Boyce J, West R, Jarvis M, Hajek P. Relapse prevention interventions for smoking cessation. Cochrane Database Syst Rev. 2019;2(2):CD003999. https://doi. org/10.1002/14651858.cd003999.pub5

10. Moyo F, Archibald E, Slyer JT. Effectiveness of decision aids for smoking cessation in adults: a quantitative systematic review. JBI Database System Rev Implement Rep. 2018;16(9):1791-1822. https:// doi.org/10.11124/jbisrir-2017-003698

11. Cigarrillo electrónico y demás ENDS. Fecha de consulta: 10 de enero, 2020. Accesible en: https://smnyct.org/informacion-general/ cigarrillo-electronico-y-demas-ends-documento-de-posicion-aamralat-ers-separ-serchile-smnyct\#attachmentsHeader

12. Grana R, Benowitz N, Glantz SA. E-cigarettes: a scientific review. Circulation. 2014;129(19):1972-1986. https://doi.org/10.1161/ circulationaha.114.007667

13. Leventhal AM, Strong DR, Kirkpatrick MG, Unger JB, Sussman S, Riggs NR, et al. Association of Electronic Cigarette Use With Initiation of Combustible Tobacco Product Smoking in Early Adolescence. JAMA. 2015;314(7):700-707. https://doi.org/10.1001/jama.2015.8950

14. Hajek P, Phillips-Waller A, Przulj D, Pesola F, Smith KM, Bisal N, et al. A randomized trial of e-cigarettes versus nicotine-replacement therapy. N Engl J Med. 2019;380(7):629-637. https://doi.org/10.1056/ nejmoa1808779

15. Lerner CA, Sundar IK, Yao H, Gerloff J, Ossip DJ, McIntosh S, et al. Vapors produced by electronic cigarettes and e-juices with flavoring induce toxicity, oxidative stress, and inflammatory response in lung epithelial cells and in mouse lung. PLoS One. 2015;10(2): e0116732. https://doi.org/10.1371/journal.pone.0116732

16. Varlet V, Farsalinos K, Augsburger M, Thomas A, Etter JF. Toxicity assessment of refill liquids for electronic cigarettes. Int $\mathrm{J}$ Environ Res Public. Health 2015;12(5):4796-4815. https://doi.org/10.3390/ ijerph120504796

Conflicto de intereses: Los autores declaran no tener conflicto de intereses. 
Anexo 1: Encuesta sobre el ENDS (Electronic Nicotine Delivery Systems) y terapia de deshabituación tabáquica.

\section{Usted está realizando una encuesta en relación a su conocimiento actual de los ENDS (Electronic Nicotine Delivery Systems) y terapia de deshabituación tabáquica sólo con fines académicos.}

Especialidad:

Si es residente, anotar grado:

1. ¿Usted utiliza el consejo breve en su consulta neumológica habitual?<smiles>C1=C[Si]=C1</smiles>

$\square$ No

En caso de que su respuesta haya sido 'NO', ¿cuál considera que es el factor por el que no utiliza el consejo breve de manera habitual en su paciente fumador?

$\square$ Conocimiento
$\square$ Tiempo
$\square$ Otro
¿Cuál?:

2. ¿Sabe usted cuál es actualmente la terapia de deshabituación de tabaco que resulta ser más efectiva para dejar de fumar?

$\square$ Sí

$\square$ No

¿Cuál?:

3. ¿Conoce los factores considerados predictores de éxito en una terapia de deshabituación de tabaco?

$\square$ Sí

$\square$ No

¿Cuáles?:

4. ¿Tiene usted conocimiento de la existencia de los ENDS para dejar de fumar?

$\square$ Sí

$\square$ No

5. ¿Usted conoce cómo funcionan en general los e-cigarettes?

$\square$ Sí

$\square$ No

6. ¿Qué tipo de ENDS conoce?

$$
\begin{aligned}
& \square \text { E-cigarrillo } \\
& \square \text { E-cigarrillo y T vapor } \\
& \square \text { E-cigarrillo, T vapor y JUULs } \\
& \square \text { Ninguno }
\end{aligned}
$$

7. ¿Usted recomienda el uso de ENDS para dejar de fumar en su práctica clínica?

$$
\begin{aligned}
& \square \text { Sí } \\
& \square \text { No } \\
& \text { ¿Por qué? }
\end{aligned}
$$

8. ¿Usted sabe qué tipos de ENDS tienen aprobación para su comercialización legal en su país?

$\square$ Sí sé

$\square$ No sé

¿Cuáles tienen aprobación para su comercialización legal en México?:

9. ¿Qué sabe usted acerca de la utilidad del uso de ENDS para dejar de fumar?

$\square$ Son terapias útiles para dejar de fumar

$\square$ Son terapias no útiles para dejar de fumar

$\square$ No conozco la utilidad del uso de ENDS para dejar de fumar

10. ¿Qué tipo de ENDS recomendaría a su paciente que consulta para dejar de fumar?

$\square$ No recomendaría ningún ENDS para dejar de fumar

$\square$ Recomendaría cualquier dispositivo acoplado a las necesidades de mi paciente, ya que existen estudios actuales que han demostrado que es mejor comparado con TRN (terapia de reemplazo de nicotina)

11. ¿Qué sabe usted acerca de la seguridad de los ENDS?

$\square$ Que son dispositivos seguros

$\square$ Que se han demostrado efectos adversos a la función respiratoria, cardiovascular e inmunológica in vitro

$\square$ Que se han demostrado efectos adversos a la función respiratoria, cardiovascular e inmunológica in vivo

$\square$ No tengo conocimiento de la seguridad de los dispositivos

12. ¿Qué sabe usted acerca de los costos de los diferentes tipos de ENDS?

$\square$ Que es más barato que un tratamiento efectivo para dejar de fumar (terapia combinada)

$\square$ Que es más caro que un tratamiento efectivo para dejar de fumar (terapia combinada)

$\square$ No tengo conocimiento sobre costos

13. ¿Usted como experto en salud respiratoria considera que su conocimiento sobre los ENDS es suficiente para recomendar o no su uso?

$\square$ Sí

14. ¿Le interesaría saber sobre de la terapia de ENDS?

$$
\begin{aligned}
& \square \text { Sí } \\
& \square \text { No } \\
& \text { ¿Por qué?: }
\end{aligned}
$$

Gracias. 